\title{
Does COVID-19 Warn Us to Revisit Virus-Induced Diabetes?
}

\author{
Muthuswamy Balasubramanyam* \\ Department of Cell and Molecular Biology, Madras Diabetes Research Foundation (MDRF), ICMR-Centre \\ for Advanced Research on Diabetes, Gopalapuram, Chennai-600086, India
}

\begin{abstract}
Diabetes is among the most frequently reported comorbidities in patients with coronavirus disease 2019 (COVID-19) and it represents a strong risk factor for developing severe, critical and fatal forms of COVID-19. The association between diabetes and worse outcome in viral infections is not unexpected, as hyperglycemia is detrimental to the control of viremia and inflammation, and very often linked to accelerated morbidity and mortality in a majority of patients. Understanding the pathophysiological mechanisms underlying the impact of diabetes on COVID-19 progression is now under critical scrutiny in several ongoing investigations, with the ultimate aim of maximizing therapeutic outcomes. On the other hand, there is a new school of thought that COVOD-19 and its devastating ravage on multiple organs could be causally linked to new-onset diabetes. Thus, the threatening new lesson is that there might be a bidirectional relationship between COVID-19 and diabetes. This review is unique in that it summarizes the very recent literature that supports why we should revisit studying virus-induced diabetes in the context of COVID-19.
\end{abstract}

\section{Introduction}

It is clearly conceived from several studies worldwide that diabetes is a significant risk factor for coronavirus disease 2019 (COVID-19) progression and several adverse endpoints, including mortality. The associations of diabetes with a variety of viruses/virus infections have been reported previously. The worldwide pandemic of COVID-19, seen in multiple races, ethnicities, age groups, and various co-morbidities, has also prompted study on whether COVID-19 infection could be causally linked to new-onset of noncommunicable diseases, particularly diabetes. Although this was a topic discussed during earlier human virus exposures, this editorial makes a humble effort to consolidate this new school of thought in the context of COVID-19, supported by very recent literature.

\section{Diabetes is a Strong Risk Factor for COVID-19 Severity}

Several studies have emphasized that individuals with co-morbid-

Keywords: COVID-19; type 1 diabetes; type 2 diabetes; SARS-CoV-2; diabetic ketoacidosis; diabetogenic; CoviDIAB.

Abbreviations: COVID-19, coronavirus disease 2019; hPSC, human pluripotent stem cell; IAPP, islet amyloid polypeptide; SARS, severe acute respiratory syndrome T1D, type 1 diabetes; T2D, type 2 diabetes; VIDM, virus-induced diabetes mellitus. Received: June 27, 2020; Revised: July 02, 2020; Accepted: July 02, 2020

${ }^{*}$ Correspondence to: Muthuswamy Balasubramanyam, Department of Cell and Molecular Biology, Madras Diabetes Research Foundation (MDRF), ICMR- Centre for Advanced Research on Diabetes, Gopalapuram, Chennai-600086, India. Tel: (9144)4396-8888; Fax: (9144)-2835-0935; E-mail: baluglobaldiab@gmail.com,

balusignal@gmail.com

How to cite this article: Balasubramanyam M. Does COVID-19 Warn Us to Revisit Virus-Induced Diabetes? Exploratory Research and Hypothesis in Medicine 2020;5(4):129-133. doi: 10.14218/ERHM.2020.00046. ities, including diabetes and cardiovascular and pulmonary diseases, have a demonstrated higher risk for severe cases of COVID-19, as well as a higher risk of mortality. ${ }^{1-3}$ Higher susceptibility of diabetic patients to virus infection was also recognized earlier. In patients with severe acute respiratory syndrome (SARS), preexisting type 2 diabetes (T2D) was shown to be independently associated with poor outcomes. ${ }^{4}$ Alqahtani et al. ${ }^{5}$ have also reported that T2D was the primary comorbidity associated with severe or lethal Middle East respiratory syndrome-coronavirus infections. A very recent multi-centric study ${ }^{6}$ reported that diabetes status increased the need for medical interventions during COVID-19 as well as increased the mortality risk of patients with COVID-19. That study also reported that good glycemic control correlated with improved outcomes in infected patients and emphasized the need for management of optimal glycemic control.

Several aspects of SARS-CoV-2 pathogenesis and potential implications for clinical management of patients with COVID-19 and diabetes as well as metabolic syndrome were also covered in recent reviews. ${ }^{7,8}$ Sardu et al. ${ }^{9}$ have also discussed the clinical and preclinical evidence supporting the hypothesis that the endothelium could be a key target organ in COVID-19, as endothelial dysfunction is linked to several co-morbidities, including diabetes and its severity. Thus, the current COVID-19 pandemic is forcing us to reconsider the ways in which effective diabetes management can be delivered during these challenging times.

\section{Is COVID-19 a Trigger for Diabetes?}

COVID-19 continues to teach us a lot of new lessons. The threatening new lesson is that there is a bidirectional relationship between COVID-19 and diabetes. Emerging evidence suggests that the coronavirus might trigger diabetes. ${ }^{10}$ Very recently, new-onset 
diabetes and severe metabolic complications of preexisting diabetes, including diabetic ketoacidosis and hyperosmolarity, have been reported in patients with COVID-19. ${ }^{11-13}$ These studies warrant that patients with elevated blood sugar and no history of diabetes should be evaluated for the possibility of new-onset diabetes mellitus and diabetic ketoacidosis, especially in the setting of concomitant COVID-19 infection.

\section{COVID-19 Warrants a Revisit on Virus-Induced Diabetes}

\section{Viruses and type 1 diabetes}

Despite the fact that mechanistic explanations for how exactly viruses may influence type 1 diabetes (T1D) etiology are still not clearly understood, viruses are considered major environmental candidates for involvement in the ontology of T1D. ${ }^{14}$ Although it is difficult to establish a cause-and-effect relationship between viral infection and diabetes in humans, preclinical animal models have provided support of such. Using BioBreeding DiabetesResistant and the LEW1.WR1 rat models, $\beta$ cell inflammation and diabetes with many similarities to the human disease have been shown to be induced by infection with the parvovirus Kilham rat virus. ${ }^{15}$ Preclinical support also comes from mouse models for virus-induced T1D, including: (a) acceleration of disease onset in prediabetic nonobese diabetic mice following coxsackievirus infection; and (b) diabetes induction by lymphocytic choriomeningitis virus infection of transgenic mice expressing viral neo-antigens under control of the rat insulin promoter. ${ }^{16}$

Apart from the animal work, the limited human studies imply that viral infection may trigger T1D. Studies that measured enterovirus RNA or viral protein found a significant association between enterovirus infection and T1D-related autoimmunity and clinical T1D. ${ }^{17}$ In the past, greater incidences of fasting glycemia and acute-onset diabetes have been reported among patients with SARS coronavirus 1 pneumonia. ${ }^{18}$ Several studies, including ones on identical twins, suggest that environmental factors such as viruses and other pathogens may be critical triggers, either through direct cytolytic effect and gradual beta cell destruction or by bystander activation of the immune system. ${ }^{19}$

\section{Viruses and type 2 diabetes}

While the development of type 2 diabetes (T2D) involves complex and heterogeneous processes, virus infection has also been implicated. Several studies have demonstrated the prevalence of T2D among persons with hepatitis, suggesting a two-way connection between diabetes and hepatitis $\mathrm{C}$ virus..$^{20,21} \mathrm{An}$ independent effect of hepatitis $\mathrm{C}$ virus - related cirrhosis on increasing T2D incidence has also been reported. ${ }^{22}$ In the context of an association of virus infection with T2D and Alzheimer's disease, ${ }^{23}$ there is also demand for identification of a single or a panel of likely bloodbased viral biomarkers for early diagnosis of diabetes as well as Alzheimer's disease. In a recent multi-omics longitudinal study, ${ }^{24}$ which followed the changes that occurred during respiratory viral infections, an impaired immune response to respiratory viral infections was demonstrated in insulin-resistant individuals and this attests a potential contribution of respiratory viral infections to the increased risk of T2D development.

In the context of COVID-19, we conceive the hypothesis that COVID-19 consequences on multiple organs, for example, hepatic manifestations as well as amyloid-associated $\beta$ cell dysfunction may drive new-onset T2D. Cases of liver damage or dysfunction (mainly characterized by moderately elevated serum aspartate aminotransferase levels) have been reported among patients with COVID-19. ${ }^{25}$ It is suggested that COVID-19 may predispose individuals to increased risk for unrecognized underlying liver disease, especially nonalcoholic fatty liver disease ${ }^{26}$ as well as other hepatic consequences of COVID-19 infection; ${ }^{27}$ these are, in turn, strong risk factors for T2D. Considering that fatty liver is an early instigator of $\mathrm{T} 2 \mathrm{D}$ and alteration in hepatic gluconeogenesis is one of the hallmarks of T2D, there is increasing attention being paid to following-up of COVID-19 patients for hepatic manifestations.

It is now well conceived that $\beta$ cell dysfunction is linked to differential activation of innate immune pathways by distinct islet amyloid polypeptide (IAPP) aggregates. ${ }^{28}$ While a study ${ }^{29}$ recently found respiratory syncytial virus and herpes simplex virus type 1 to accumulate a rich and distinctive protein corona in different biological fluids, it is interesting to note that the study also demonstrated that viruses bind amyloidogenic peptides in their corona and catalyze amyloid formation via surface-assisted heterogeneous nucleation. Very recently, using a vaccine based on virus-like particles coupled to IAPP peptides (that induce specific antibodies against aggregated IAPP), it was demonstrated in vivo that the vaccine prevented IAPP depositions, decreased pro-inflammatory cytokines, and delayed onset of hyperglycemia. ${ }^{30}$ These collective findings emphasize that the mechanistic convergence between viral and amyloid pathologies should be studied in-depth in the context of T2D and Alzheimer's disease.

\section{Diabetogenic effect of COVID-19?}

There is now a new school of thought, hypothesized by Rubino et $a l .{ }^{31}$ that there might be a potential diabetogenic effect of COVID-19. However, the following questions remain: a) Are the alterations of glucose metabolism that occur with a sudden onset in severe COVID-19 persistent or transient? b) Will it remit when the infection resolves? c) How frequent is the phenomenon of new-onset diabetes, and is it a classical type 1 or type 2 diabetes, or a substratified new type of diabetes? and d) Do these patients remain at higher risk for diabetic ketoacidosis and other complications?

Other interesting questions I propose are: a) If hyperglycemia in COVID-19 patients is transient, will it pose a potential risk for later T2D development in a subset of patients, like the phenomenon that is very well established in gestational diabetes mellitus? b) Will COVID-19 infection exploit individuals with prediabetes for early development of T2D?

Answering these questions will require frequent monitoring and follow-up in COVID-19 patients subsequent to their recovery.

\section{Proposing "CoviDIAB" for COVID-19 follow-up}

The good news is that brain-storming by an international group of leading diabetes researchers led to establishment of a global registry of patients with COVID-19-related diabetes, called "CoviDIAB" (covidiab.e-dendrite.com). ${ }^{31}$ The major goal of the registry is to determine the extent and phenotype of new-onset diabetes that is defined by hyperglycemia in confirmed COVID-19 patients (with negative history of diabetes and normal glycated hemoglobin level). It is mentioned that the registry will be expanded to include patients with preexisting diabetes who present with severe acute metabolic disturbance(s), so as to investigate the epidemiologic features and pathogenesis of COVID-19-related diabetes and to 


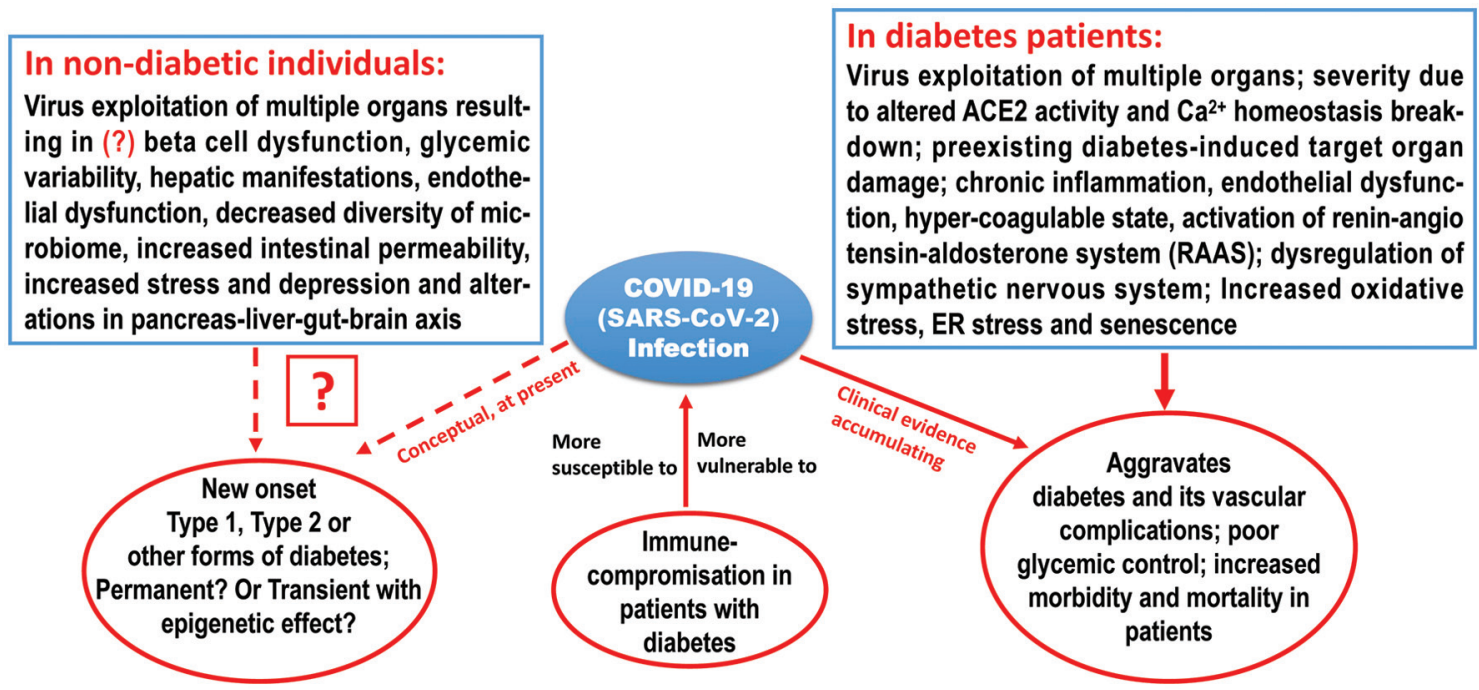

Fig. 1. The two-way relationship between COVID-19 and diabetes.

gain insights on the optimal management of patients during and after the course of COVID-19. Follow-up on COVID-19 patients for diabetes incidence would also stimulate conduct of experimental biology research using human pluripotent stem cells (hPSCs) and organoids.

In fact, Yang et al. ${ }^{32}$ generated a library of hPSC-derived cells/ organoids and showed that hPSC-derived hepatic and pancreatic cells are permissive to SARS-CoV-2 infection. This fascinating observation was further validated using adult primary human islets, adult hepatic and cholangiocyte organoids, and a humanized mouse model. Thus, closer monitoring of individuals with high risk for diabetes is the need of the day, to evaluate the contribution of SARS-CoV-2 in previously infected COVID-19 patients regarding progression toward type 1 or type 2 diabetes. It is expected that such hPSC/organoid-based platform can also be used for drug screening, with an aim towards development of prospective antiviral therapeutics.

\section{Future directions}

As COVID-19 pandemic continues, there is an imperative need for COVID-19 follow-up studies to track and understand its longterm health consequences. It is important to notice that there are few cases of reactivation of COVID-19 in apparently cured patients and such relapse of COVID-19 could pose a vicious cycle of public health burden. Although preliminary evidences suggest that antibody responses occur in those who have been infected, further studies are needed to differentiate whether these IgG antibodies are protective or not $\&$ if protective, how long this protection lasts needs to be evaluated. Earlier follow-up studies done in patients infected with SARS and MERS-CoV did noticed long-term health burden with poor quality of life. Therefore, depending on the individual's metabolism and immunity, there is also a concern in that your health may never be the same after COVID-19 infection and in this context, several cohort studies are underway to study the long-term health consequences. The proposed 'CoviDIAB' study discussed in this review is one such visionary effort with regard to studying the genesis of diabetes and its complications. In fact, COVID-19 follow-up studies should track the occurrence of all non-communicable diseases to better understand the long-term health consequences. Such cohort studies that follow populations over years are the need of the day to trace the pandemic's physical, mental and social consequences so as to develop and adopt suitable prevention and disease-management strategies.

\section{Conclusions}

As more and more studies are being conducted and published, there is increasing attention in studying the bidirectional relationship between COVID-19 and diabetes - nut-shell details are illustrated in Figure 1. First, it is now well-conceived that diabetes patients, characterized by their subclinical inflammation and immune-compromised state, are more susceptible/vulnerable to COVID-19 infection. Secondly, COVID-19 infection in diabetic patients aggravates morbidity and may be linked to increased mortality. The biological explanations for this could be virus exploitation of multiple organs, severity due to altered ACE2 activity and $\mathrm{Ca}^{2+}$ homeostasis breakdown, preexisting diabetes-induced target organ damage, chronic inflammation, endothelial dysfunction, hyper-coagulable state, activation of renin-angiotensin-aldosterone system, and dysregulation of the sympathetic nervous system. However, there is caution in that all these mechanistic explanations are based on findings from retrospective studies that reviewed COVID-19 patients' records, which do not provide evidence as strong as the results of controlled clinical trials. Again, the good news is that several clinical trials on COVID-19 patients are presently under way to test and increase our understanding on pathogenesis. Thirdly, diabetogenic effects of COVID-19 represents a totally a new school of thought, originating from the in-depth observations on COVID-19 patients from December 2019 to date. It is hypothesized that COVID-19 could trigger diabetes and could occur due to virus exploitation of multiple organs resulting in (possibly) $\beta$ cell dysfunction, glycemic variability, hepatic manifestations, endothelial dysfunction, decreased diversity of microbiome, increased intestinal permeability, increased stress and depression, and alterations in the pancreas-liver-gut-brain axis in non-diabetic individuals. Biological explanations for the above are at present conceptual and the rightly planned COVID-19 follow-up studies, 
such as 'CoviDIAB', should deliver mechanistic outcomes in this direction.

It appears that understanding how COVID-19-related diabetes develops and the new-biology etiology of this disease would pave the way for substratification of diabetes, perhaps using a special category which I proactively propose as "VIDM" for virus-induced diabetes mellitus. Such substratification of diabetes might require different treatment modalities in the context of personalized/precision medicine. With the alarming pandemic of COVID-19, it's time for India and other developing countries to join the global registry as well as to start their own COVID-19 registry with much more focus on cohort-specific follow-up investigations.

It is expected that this brief overview would stimulate more brain-storming and discussion on the mechanisms and possibilities of COVID-19-induced diabetes among the researchers, clinicians, government health authorities and patients.

\section{Acknowledgments}

The author gratefully acknowledges general career support by the Indian Council of Medical Research (ICMR) \& Department of Biotechnology (DBT), Government of India.

\section{Funding}

No dedicated source of funding.

\section{Conflict of interest}

The author declares having no conflicts of interest related to this publication.

\section{References}

[1] Wang D, Hu B, Hu C, Zhu F, Liu X, Zhang J, et al. Clinical characteristics of 138 hospitalized patients with 2019 novel coronavirus-infected pneumonia in Wuhan, China. JAMA 2020;323(11):1061-1069. doi:10.1001/jama.2020.1585.

[2] Zhang JJ, Dong X, Cao YY, Yuan YD, Yang YB, Yan YQ, et al. Clinical characteristics of 140 patients infected with SARS-CoV-2 in Wuhan, China. Allergy 2020. doi:10.1111/all.14238.

[3] Zhou F, Yu T, Du R, Fan G, Liu Y, Liu Z, et al. Clinical course and risk factors for mortality of adult inpatients with COVID-19 in Wuhan, China: a retrospective cohort study. Lancet 2020;395:1054-1062. doi:10.1016/S0140-6736(20)30566-3.

[4] Yang JK, Feng Y, Yuan MY, Yuan SY, Fu HJ, Wu BY, et al. Plasma glucose levels and diabetes are independent predictors for mortality and morbidity in patients with SARS. Diabet Med 2006;23(6):623-628. doi:10.1111/j.1464-5491.2006.01861.x.

[5] Alqahtani FY, Aleanizy FS, Ali El Hadi Mohamed R, Alanazi MS, Mohamed N, Alrasheed MM, et al. Prevalence of comorbidities in cases of Middle East respiratory syndrome coronavirus: a retrospective study. Epidemiol Infect 2018;147:1-5. doi:10.1017/S0950268818002923.

[6] Zhu L, She ZG, Cheng X, Qin JJ, Zhang XJ, Cai J, et al. Association of Blood Glucose Control and Outcomes in Patients With COVID-19 and Pre-existing Type 2 Diabetes. Cell Metab 2020;31(6):1068-1077.e3. doi:10.1016/j.cmet.2020.04.021

[7] Hussain A, Bhowmik B, do Vale Moreira NC. COVID-19 and diabetes: Knowledge in progress. Diab Res Clin Pract 2020;162:108142. doi:10.1016/j.diabres.2020.108142.

[8] Bansal R, Gubbi S, Miniyappa R. Metabolic Syndrome and COVID 19:
Endocrine-Immune-Vascular Interactions Shapes Clinical Course. Endocrinology 2020:bqaa112. doi:10.1210/endocr/bqaa112.

[9] Sardu C, Gambardella J, Morelli MB, Wang X, Marfella R, Santulli G. Hypertension, Thrombosis, Kidney Failure, and Diabetes: Is COVID-19 an Endothelial Disease? A Comprehensive Evaluation of Clinical and Basic Evidence. J Clin Med 2020;9(5):1417. doi:10.3390/jcm9051417.

[10] Mallapaty S. Mounting Clues Suggest the Coronavirus Might Trigger Diabetes. Nature 2020;583(7814):16-17. doi:10.1038/d41586-02001891-8.

[11] Chee YJ, Ng SJH, Yeoh E. Diabetic ketoacidosis precipitated by Covid-19 in a patient with newly diagnosed diabetes mellitus. Diabetes Res Clin Pract 2020;164:108166. doi:10.1016/j.diabres.2020.108166.

[12] Li J, Wang X, Chen J, Zuo X, Zhang H, Deng A. COVID-19 infection may cause ketosis and ketoacidosis. Diabetes Obes Metab 2020. doi:10.1111/dom.14057.

[13] Heaney Al, Griffin GD, Simon EL. Newly diagnosed diabetes and diabetic ketoacidosis precipitated by COVID-19 infection. Am J Emerg Med 2020:S0735-6757(20)30488-5. doi:10.1016/j.ajem. 2020.05.114.

[14] Schneider DA, von Herrath MG. Potential viral pathogenic mechanism in human type 1 diabetes. Diabetologia 2014;57(10):20092018. doi:10.1007/s00125-014-3340-7.

[15] NeedellJC,Zipris D. RatModels ofVirus-Induced Type1 Diabetes. Methods Mol Biol 2020;2128:107-114. doi:10.1007/978-1-0716-03857_8.

[16] Christoffersson G, Flodstorm-Tullberg M. Mouse Models of VirusInduced Type 1 Diabetes. Methods Mol Biol 2020;2128:93-105. doi:10.1007/978-1-0716-0385-7_7.

[17] Yeung WC, Rawlinson WD, Craig ME. Enterovirus infection and type 1 diabetes mellitus: systematic review and meta-analysis of observational molecular studies. BMJ 2011;342:d35. doi:10.1136/bmj.d35.

[18] Yang JK, Lin SS, Ji XJ, Guo LM. Binding of SARS coronavirus to its receptor damages islets and causes acute diabetes. Acta Diabetol 2010;47(3):193-199. doi:10.1007/s00592-009-0109-4.

[19] Rodriguez-Calvo T, Sabouri S, Anquetil F, von Herrath MG. The Viral Paradigm in Type 1 Diabetes: Who Are the Main Suspects? Autoimmun Rev 2016;15(10):964-969. doi:10.1016/j.autrev.2016.07.019.

[20] Mehta SH, Brancati FL, Sulkowski MS, Strathdee SA, Szklo M, Thomas DL. Prevalence of type 2 diabetes mellitus among persons with hepatitis C virus infection in the United States. Ann Intern Med 2000;133(8):592-599. doi:10.7326/0003-4819-133-8-200010170-00 009.

[21] Hammerstad SS, Grock SF, Lee HJ, Hasham A, Sundaram N, Tomer Y. Diabetes and hepatitis C: a two- way association. Front Endocrinol (Lausanne) 2015;6:134. doi:10.3389/fendo.2015.00134.

[22] Li J, Zhang T, Gordon SC, Rupp LB, Trudeau S, Holmberg SD, et al. Impact of sustained virologic response on risk of type 2 diabetes among hepatitis $\mathrm{C}$ patients in the United States. J Viral Hepat 2018;25(8):952-958. doi:10.1111/jvh.12887.

[23] Karim S, Mirza Z, Kamal MA, Abuzenadah AM, Azhar El, Al-Qahtani $\mathrm{MH}$, et al. An Association of Virus Infection with Type 2 Diabetes and Alzheimer's Disease. CNS Neurol Disord Drug Targets 2014;13(3):429-439. doi:10.2174/18715273113126660164.

[24] Zhou W, Sailani MR, Contrepois K, Zhou Y, Ahadi S, Leopold SR, et al. Longitudinal multi-omics of host-microbe dynamics in prediabetes. Nature 2019;569(7758):663-671. doi:10.1038/s41586-019-1236-x.

[25] Amin M. COVID-19 and the liver: overview. Eur J Gastroenterol Hepatol 2020. doi:10.1097/MEG.0000000000001808.

[26] Testino G. Covid-19 Infection, Liver Injury and Prognosis: A Suggestion. Eur J Gastroenterol Hepatol 2020. doi:10.1097/MEG. 0000000000001809.

[27] Portincasa P, Krawczyk M, Machill A, Lammert F, Di Ciaula A. Hepatic Consequences of COVID-19 Infection. Lapping or Biting? Eur J Intern Med 2020;77:18-24. doi:10.1016/j.ejim.2020.05.035.

[28] Westwell-Roper C, Denroche HC, Ehses JA, Verchere CB. Differential Activation of Innate Immune Pathways by Distinct Islet Amyloid Polypeptide (IAPP) Aggregates. J Biol Chem 2016;291(17):8908-8917. doi:10.1074/jbc.M115.712455.

[29] Ezzat K, Pernemalm M, Pålsson S, Roberts TC, Järver P, Dondalska A, et al. The viral protein corona directs viral pathogenesis and amyloid aggregation. Nat Commun 2019;10(1):2331. doi:10.1038/s41467- 
019-10192-2.

[30] Roseti ES, Boyle CN, Zeman DT, Sande-Melon M, Storni F, CabralMiranda G, et al. Vaccination Against Amyloidogenic Aggregates in Pancreatic Islets Prevents Development of Type 2 Diabetes Mellitus. Vaccines (Basel) 2020;8(1):116. doi:10.3390/vaccines8010116.

[31] RubinoF, AmielSA, ZimmetP, AlbertiG, Bornstein S, Eckel RH, etal. New-
Onset Diabetes in Covid-19. N Engl J Med 2020. doi:10.1056/NEJMc 2018688.

[32] Yang L, Han Y, Nilsson-Payant BE, Gupta V, Wang P, Duan X, et al. A Human Pluripotent Stem Cell-based Platform to Study SARS-CoV-2 Tropism and Model Virus Infection in Human Cells and Organoids. Cell Stem Cell 2020;27(1):125-136.e7. doi:10.1016/j.stem.2020.06.015. 